ISSN 1112-9867

\title{
LIGHTING AND ENERGY SUPPLY FOR HEATING IN BUILDING USING ALGAE POWER
}

\author{
F. Sepehri
}

Researcher, lecturer of Architecture at Soore University Chapter,

Published online: 15 May 2016

\begin{abstract}
Today, given the rapid population growth and human needs as well as the increasing consumption of energy, the phenomenon of energy crisis can be seen in the world. In the past one hundred years, the need for sustainable, cheap and clean fuel sources has been taken into consideration more than before. A significant proportion of this energy is consumed in buildings. Therefore, access to clean and healthy energy in buildings can have a significant role in improving environmental conditions. This article wants to present the definition and the usage of one of the energy resources made by nanotechnology and its effect on sustainable architecture. This kind of clean energy is obtained from algae sources (algae and microalgae). In recent years, numerous studies have been conducted on the use of algae as raw materials for biofuel production. In this article, the method of producing energy from these algae along with their application in sustainable architecture has been investigated. For this purpose, a few examples of algal energy uses in the exterior and interior spaces of building and forest canopies have been introduced.
\end{abstract}

Key words: energy, algae, lighting, nano, architecture

\footnotetext{
Author Correspondence, e-mail: fa_sepehri@yahoo.com

doi: http://dx.doi.org/10.4314/jfas.v8i3s.13
}

Journal of Fundamental and Applied Sciences is licensed under a Creative Commons Attribution-NonCommercial 4.0 International License. Libraries Resource Directory. We are listed under Research Associations category, 


\section{INTRODUCTION}

During the $19^{\text {th }}$ and $20^{\text {th }}$ centuries, man has learned how to use the focused energies such as fossil fuel. With industrial development and increase in production, some problems have arisen on the planet, and have become more serious day by day. An increase in disease and mortality caused by environmental pollution, acid rains and destruction of ecosystems and ozone are some of the consequences. It's the long years that scientists and researchers have done a lot of activities to make use of algae as an energy source. Algae that grow quickly and are able to absorb threequarters of the light emitted from the sun have a lot of energy and researchers try to convert this energy into other forms and operate it.

Advances in nanotechnology increasingly give us the possibility to be able to take practical advantage of plants in energy production and its absorption. Latro lamp is one of the products that can use the seaweed plant as a source of energy and will be used by the public in the future. (Mike Thomson, 2012)

The idea of this scientific breakthrough has been raised by scientists from Stanford University. The lamb supplies its energy to kindle using sunlight, carbon dioxide and water.

Another product that is produced from seaweed can be mentioned as the project of "BIQ" first building with green bioreactor façade, which has been introduced as an example of the renewable energy concept around the world. The facade of this building is formed by bioreactors in double glass plates, microalgae are growing in glass facade that produce heat through photosynthesis and biomass by using the temperature of the sun, as well as usually are considered as sound insulation. Some believe the facade of the building is somewhat similar to an exhibition of flowers and plants, but in principle, this architecture is not nothing but a vertical farm for growing algae. Designers of the building are fully committed to renewable energies. This pioneer system of energy production can achieve biofuel through algae cultivation, which produces heat and cause the shade and keep the building from the street noise. Perhaps now, the use of algae as a handmade energy source seems to be a dream, but it will become commonplace in the next 10 years. (Rainer Mueller, 2013)

Another new perspective that further specifies the use of algae is ecological item, which is a combination of several construction techniques that utilizes a variety of materials and benefits from the properties of algae. These factors predict sustainable opportunities for a canopy or shelter for humans in the nature. Of course, this design not only are responsive in the use of algae 
in municipal areas, but it can have a significant impact on human use of nature, forest and natural open spaces, and make them use wider and more comfortable.

\section{Nano Green Architecture $=$ Nano green technology + Architecture}

Nano green architecture has two purposes: production of Nano materials without harming the environment and developing products that directly and indirectly benefit the environment. Green Nanotech based monitors the production in order to minimize the unforeseen negative consequences and predict the ecological basis of products in the total life cycle. Nanotechnology is entirely based on more performance in less space. It is noted in the upcoming three principles: (Mousavi, Noor Almahdy and Razavi, M. 2011)

A) Nanotechnology and clean technology: Every new technology is expensive at first, but after mass production leads to save after construction costs.

B) Renewable energies: It concerns about the quest for replacing nonrenewable fuels with renewable fuels and energy.

C) Confront the reality of using nanotechnology has enormous potential in various fields, including Nano green architecture, as long as the conclusion of researchers' studies be based on renewable energy resources to be able to use the studies practically and based on the realities of the industry.

\section{(Latro Lamp)}

In Latin, Latro means thief but here refer to the concept of using electrons from photosynthesis of algae). The potential of this species of algae is in such a way that can easily switch a lamp on. To do so, you can put the algae inside a bulb and create light in a very simple way. Since Latro is a life giving and respiratory product, foster of algae is very easy and it only needs sunlight, carbon dioxide $(\mathrm{CO} 2)$ and water, thereby it provides a very simple way to produce energy. There are some algae inside the lamp that can produce energy using the carbon dioxide that is in the human's expiration. You can easily locate Latro lamp in the sun exposure and blew into it to turn it on! Researchers have found that an electric current can be extracted from algae photosynthesis. This lamp is equipped with a solar battery that stores solar energy during the day so it can be used even when sunlight is not available. These lamps do not need to have a groove or slot, and water and a system are used to transport carbon dioxide gas to the lamps. (image 1) 

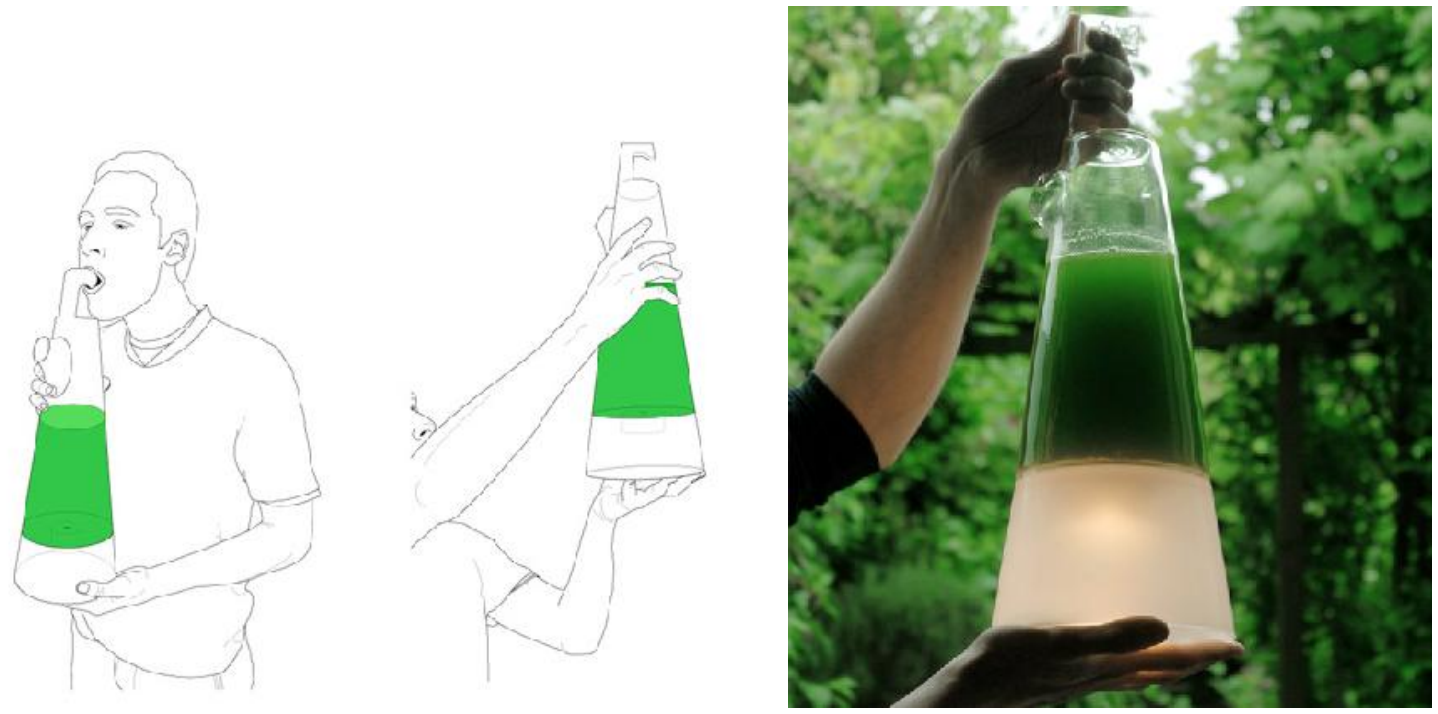

Maintenance of algae on the glass and in front of the light and blowing to lighting image (1)

\section{Photosynthetic Furniture: Living Fixtures Produce Light \& Heat}

Employing natural processes to illuminate and warm interior spaces, this series of algaebased furnishings combines beautiful glass vessels and organic matter to create lovely yet functional home objects. Each fixture generates light, heat and oxygen using methods similar to those seen in bio-adaptive architectural facades.

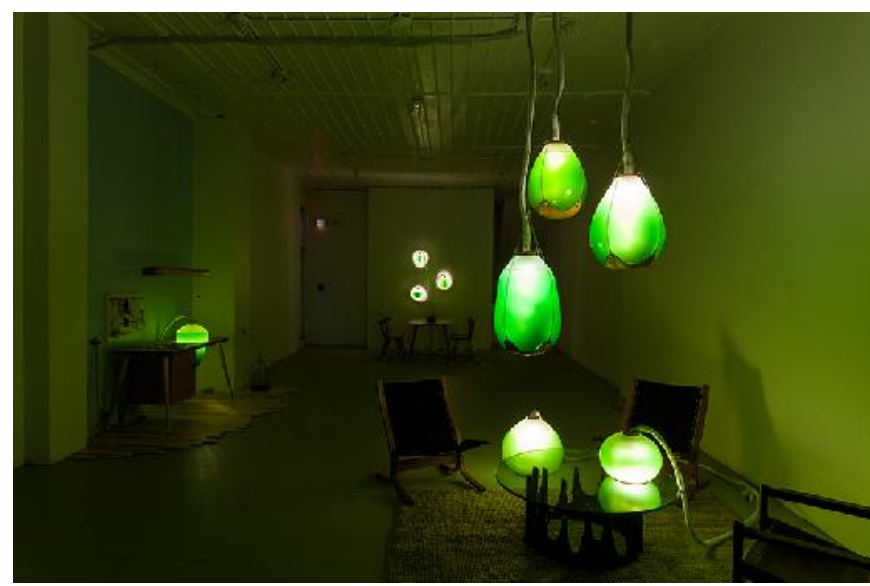

Courtesy of the mattress factory Aug 06,2015 Image (2)

Designed by Jacob Douenias and Ethan Frier, objects in the Spirulina series utilize cyanobacteria, "chosen for its rich green hue, light absorbency, and culinary qualities," set in alkaline water and housed in shapely glass vessels. Found all over the world, this versatile 
algae can survive and thrive in all kinds of environments and is already widely used in other forms of biotechnology. In this commissioned series, a set of spaces show off the various ways these organic home furnishings can be deployed in living and dining areas, popping up from tables, sprouting from walls, sitting on the floor or hanging from the ceiling. The various units are linked by a half mile of hidden plumbing and wiring and controlled via a concealed computer system that both analyzes results and controls each appendage

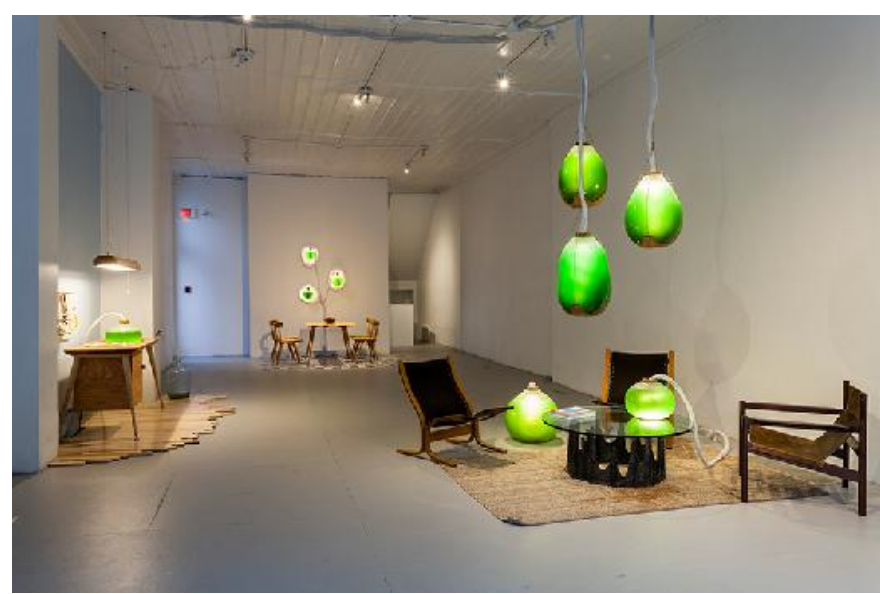

Courtesy of the mattress factory Aug 06,2015 image(3)

Living Things is now installed at the Mattress Factory Museum of Contemporary Art in Pittsburgh, Pennsylvania until March 27, 2016. While these experimental installations remain artistic prototypes for now, they and other similar endeavors continue to explore the range of near-future applications for organic matter in everyday domestic settings and built environments. (image 2,3 ) 

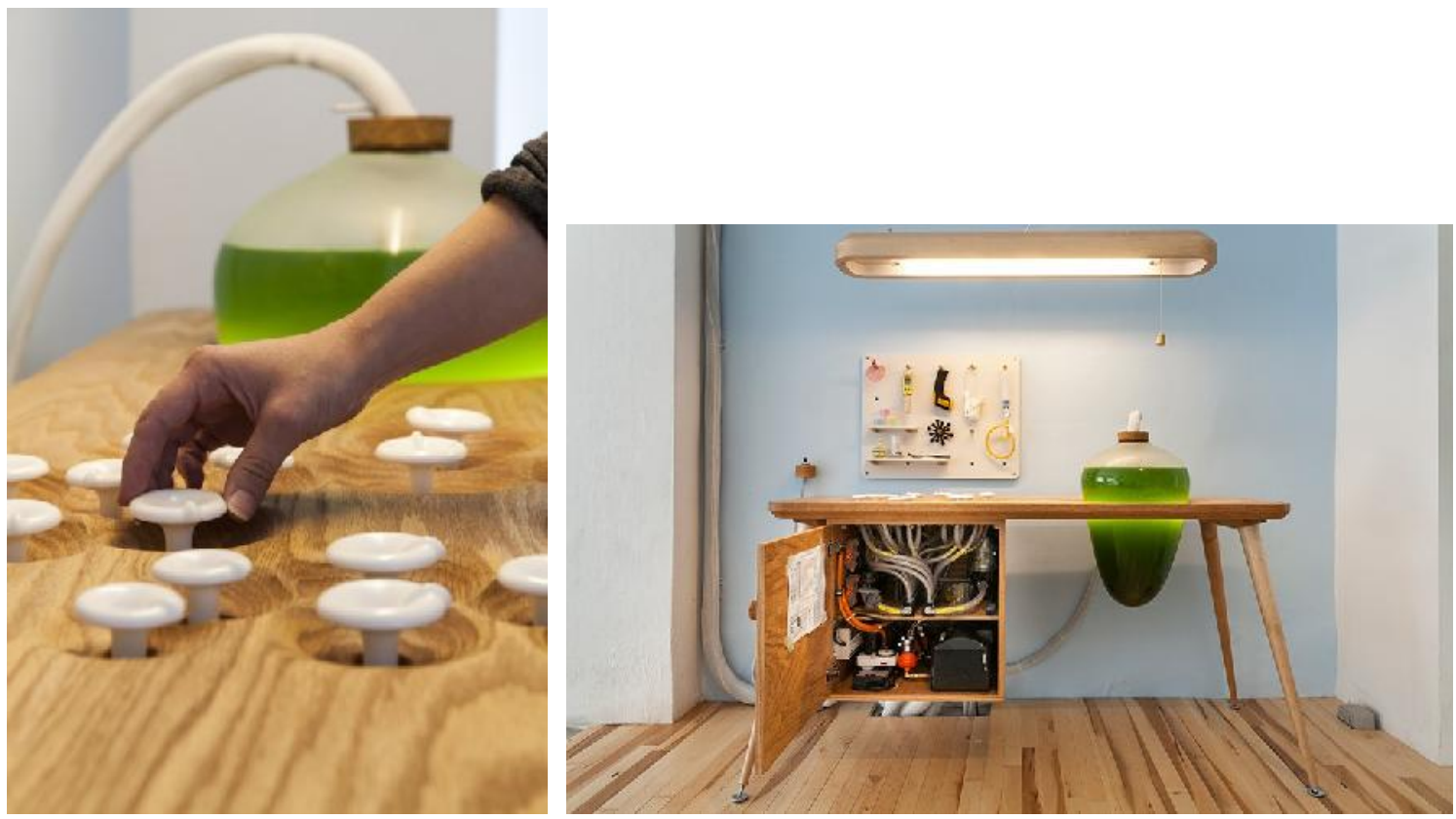

6/2015 provided by the mattress factory image(5) Display illumination system operation by algae image (4)

But how does it work? "The 3D printed nylon knobs embedded in the surface of this workstation actuate eighteen valves which allow for the harvesting of Spirulina when the culture becomes dense enough, and the supply of fresh liquid media to each vessel. Inside the cabinet the pumps, tubing, manifolds, LED drivers, air pumps, heater connections and filters which comprise the heart of the life support system"(image 4,5 )

More from its creators: "The morphologies of hand-blown glass vessels function both as lighting and heating elements for the human occupants, and high functioning photobioreactors which provide heat, light, agitation, air supply, nutrient and waste control to the living algae inside." (image 6,7 ) 

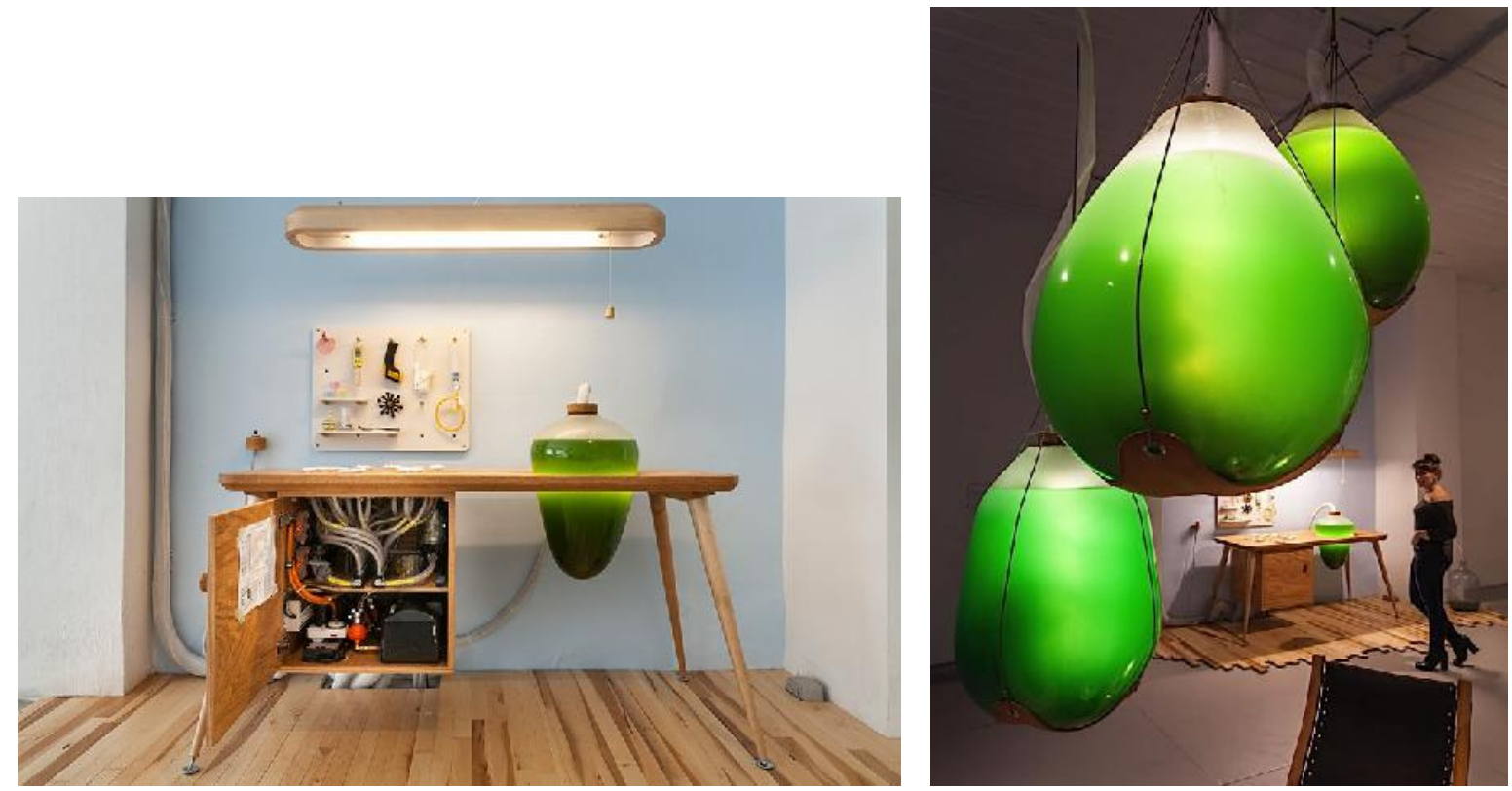

Aug/ 6/ 2015 provided by the mattress factory (image 6,7 )

\section{Algae-Fueled Building: World's First Bio-Adaptive Façade}

Bio-reactors and micro-algae sound like the stuff of science fiction, but this is the real deal: biomass built into panel glass generates heat and power, also acting as a responsive light and sound barrier, all in one brilliant new building in Hamburg. These same technologies are poised to forever change how we think about and approach the relationship between nature's organic materials and humankind's architectural creations in the future.

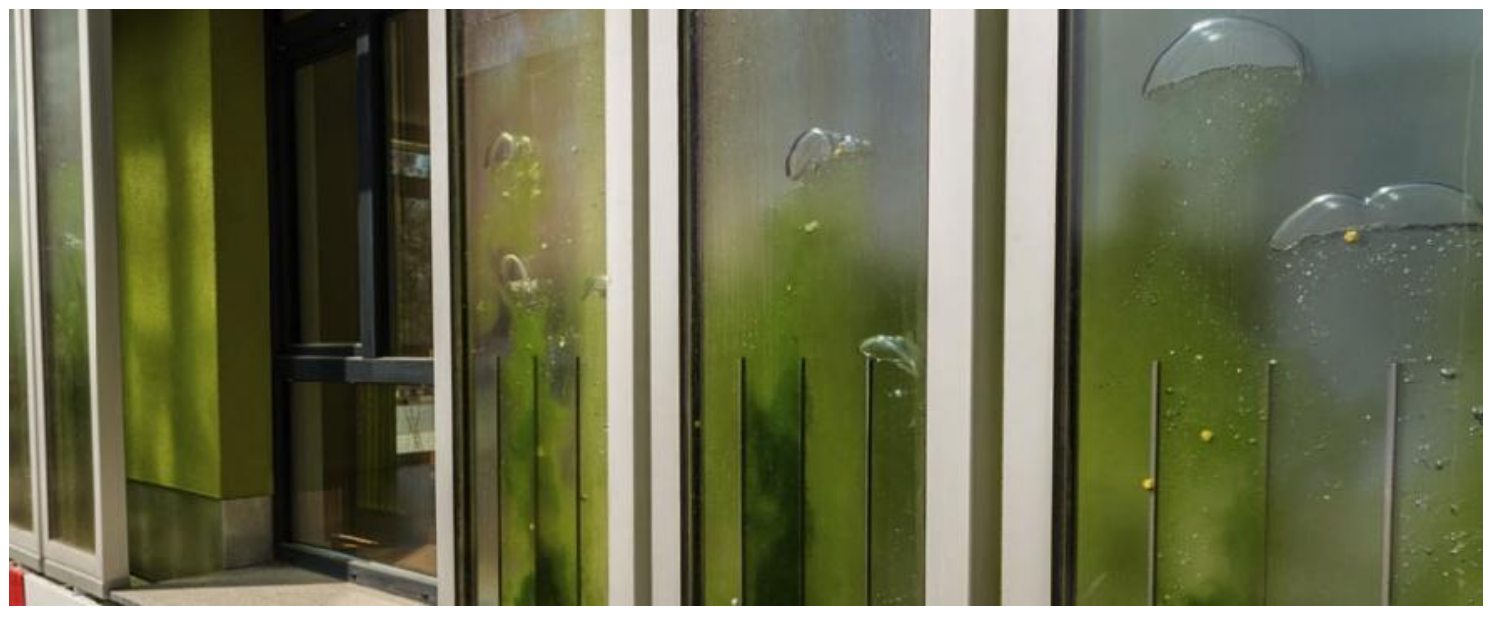

A close view of the Bio-reactors in the building (image 8) 
Arup has long been predicting incredible innovations in architecture, but they are also keen to show that their designers and engineers are actually working toward world-changing technologies - hence BIQ House, constructed in conjunction with Splitterwerk
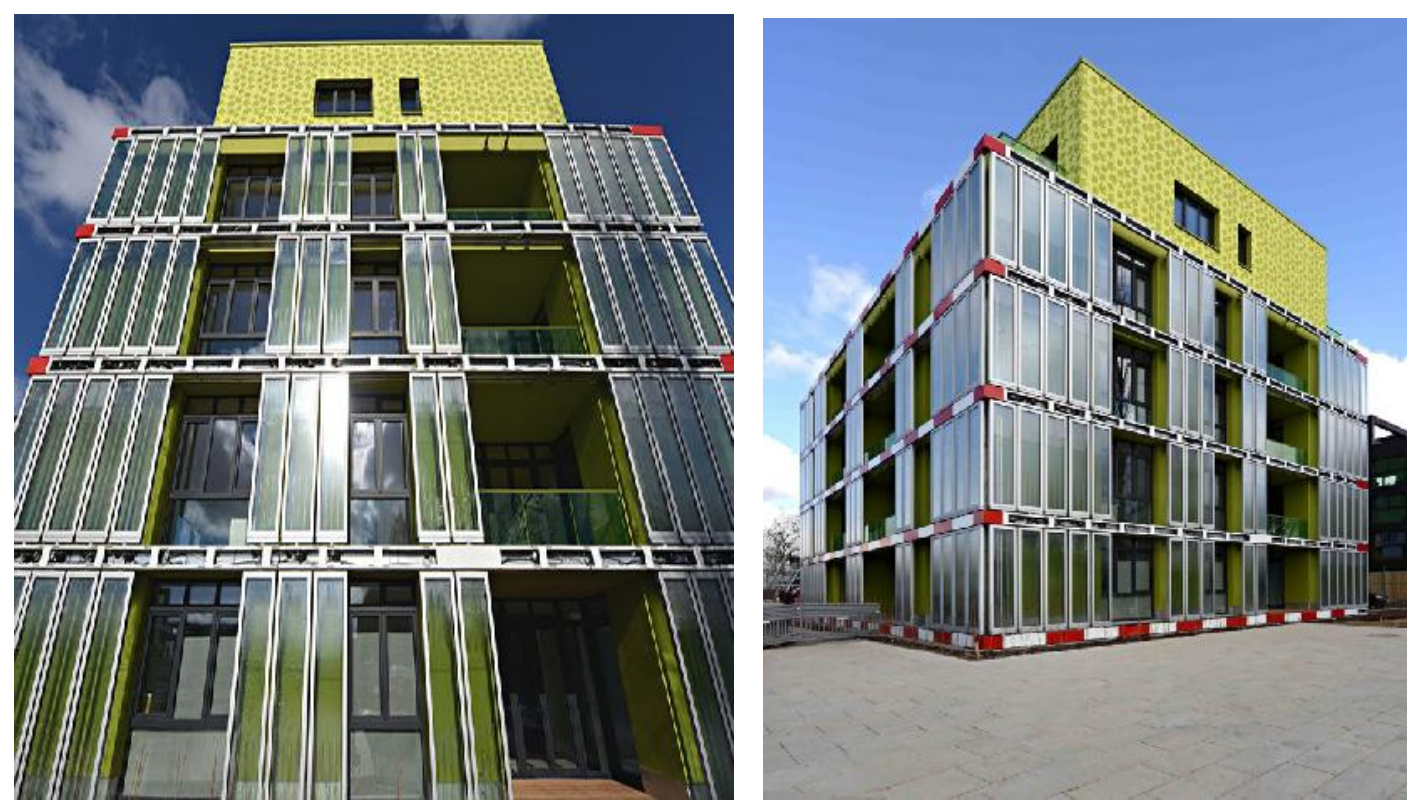

Apr 12, 2013 two sides of the building are clad in panels containing live micro-algae image $(9,10)$

Bright sunlight causes the bio-reactors to grow faster and supply more shade on demand. The resulting biomass captures solar heat as well, and can be harvested and used as a source of energy itself. In essence, it is an architectural ecosystem in which all parts of the process are not only sustainable but multi-functional and fully integrated. (image 9,10 ) 


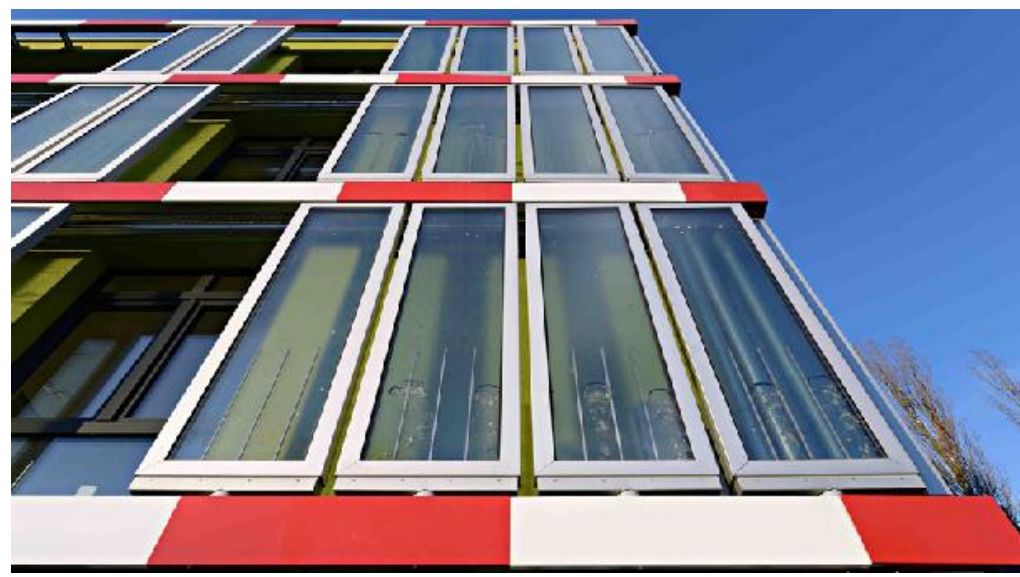

Glass photo-bioreactors create the suitable environment for photosynthesis courtesy arup/splitterwerk image (11)

bioreactors, are clear containers that create a controlled environment for an algae farm. Exposed to sunlight, the algae photosynthesise, absorbing $\mathrm{CO} 2$ as they grow. Nutrients and $\mathrm{CO} 2$ are circulated through the bioreactors to encourage growth. Periodically, the algae are collected and fermented in a nearby biomass plant, then burned to produce electricity. (image 8)

The BIQ building, which contains 15 apartments, opened its doors in April. Its main source of energy is heat recovery, capturing energy not used in photosynthesis from the bioreactor solution. The façade also acts as a natural thermostat: thick algae growth in the summer keeps the sunlight out. (image11)

\section{The proliferation and growing of algae:}

This is usually done in two ways:

- Use of open pools

- Use of closed photo-bioreactors

Pool-phase method is a simple and inexpensive method volume of 100 liters to 10 billion liters this pool can vary. The pools open in simplicity and economy also faced with difficulties and problems. One of the problems with penetration and growth of unwanted algae species and inappropriate at the pool there is another disadvantage due to the open pool, a large part of the transferred gas cannot escape. (Amir Omidvar, 2014)

In the photo-bioreactors closed almost solved the above-mentioned disadvantages and in view of a photo-bioreactor you see in the image below. The only disadvantage of this method is relatively higher costs than the pool is open. (Amir Omidvar, 2014) 

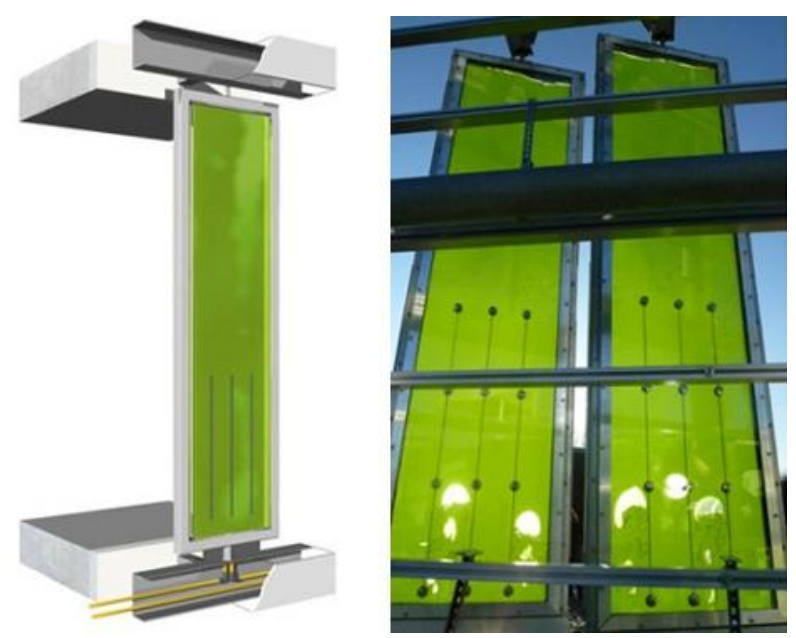

prototype of the algae-filled glass louvers arup (C) splitterwerk image(12)

\section{Bioreactor}

Bioreactors tanks are transparent and controlled environment for the phenomenon of photosynthesis. (Warm, John) algae are exposed to sunlight, through photosynthesis during plant growth, absorb carbon dioxide. The solution in these Bioreactors; (biological reactors), nutrients and carbon dioxide are working to improve the growth of the algae. The compressed air is pumped into the tanks to accelerate algae growth and also prevent them from settling and perishable. To clean the glass, the blades used to automatically do this. The glass surfaces can double the solar collectors, heat absorbing. "It is part of sunlight that is not absorbed by algae, causing heat. This heat can be used for heating buildings or water based geothermal system in the basement of the building be saved". (Warm, John)

Occasionally algae are collected and stored in tanks. Energy purchased the algae to ferment them in a place near the building. The product of this process, methane gas is used to generate electricity. "This method of generating electricity, not creating problems for the environment and not aggravate global warming. Complexes with 15 or more units can also accommodate the plant and electricity use. "(Warm)

An example of bioreactor is used B.I.G building exterior is covered in two aspects of the project bioreactor, the bioreactor is the world's first full view. The building did not believe the so-called Form victims of the use or performance. "The green color of chlorophyll in the algae mixed with 
bubbles of compressed air and a view like decorative lava lamps associated with it. Prior to this, many people doubted that the same can be released facade, but now those doubts have been removed". (Warm)

The fumes from the cars on freeways can be used as a source of carbon dioxide to produce algae. The more carbon dioxide, more lipids in algae production and can obtain more energy. (Landrono, Annika)

"We have about bacterial contamination and finding suitable species that grow quickly and produce more extractable lipid, more studies must be done." (Valzak, Scott) cost, another obstacle is the growth of algae technology. It is estimates that the cost per kilowatt hour of algal bioreactors, about seven times and 14 times the solar power is electricity produced from petroleum. (Valzak) (image 12)

The efficiency of the conversion, the light energy is converted to the view that, for biogas for heating $10 \%$ and $38 \%$ of that total is about $50 \%$. In the future, more research will be done on this issue. (This is in contrast to conventional photovoltaic systems efficiency is $15 \%$ ) Solar panels installed on the roofs and underground thermal storage system supplements can Bioreactors; energy and heat are generated. The creators claim that it can be provided through one hundred percent of the energy needs of the building. The only problem is the development and adoption of this technology, the high cost of its production. (Kroners, Martin) (image 13)

It is estimated that $75 \%$ of the population to 9 billion over the next fourteen nations will live in urban areas and dense urban environments of the organisms living in these conditions should be used to meet part of their energy. It is worth noting the company before this concept of skyscrapers 2050 has to offer; this plan includes building alive with algae is that a module is flexible, with the air purification system, sites, food production and green space inside is that maintenance is by robots. Smart systems are the future of brain structures, management and use of various sources of energy provided by residents. (Warm, John) 


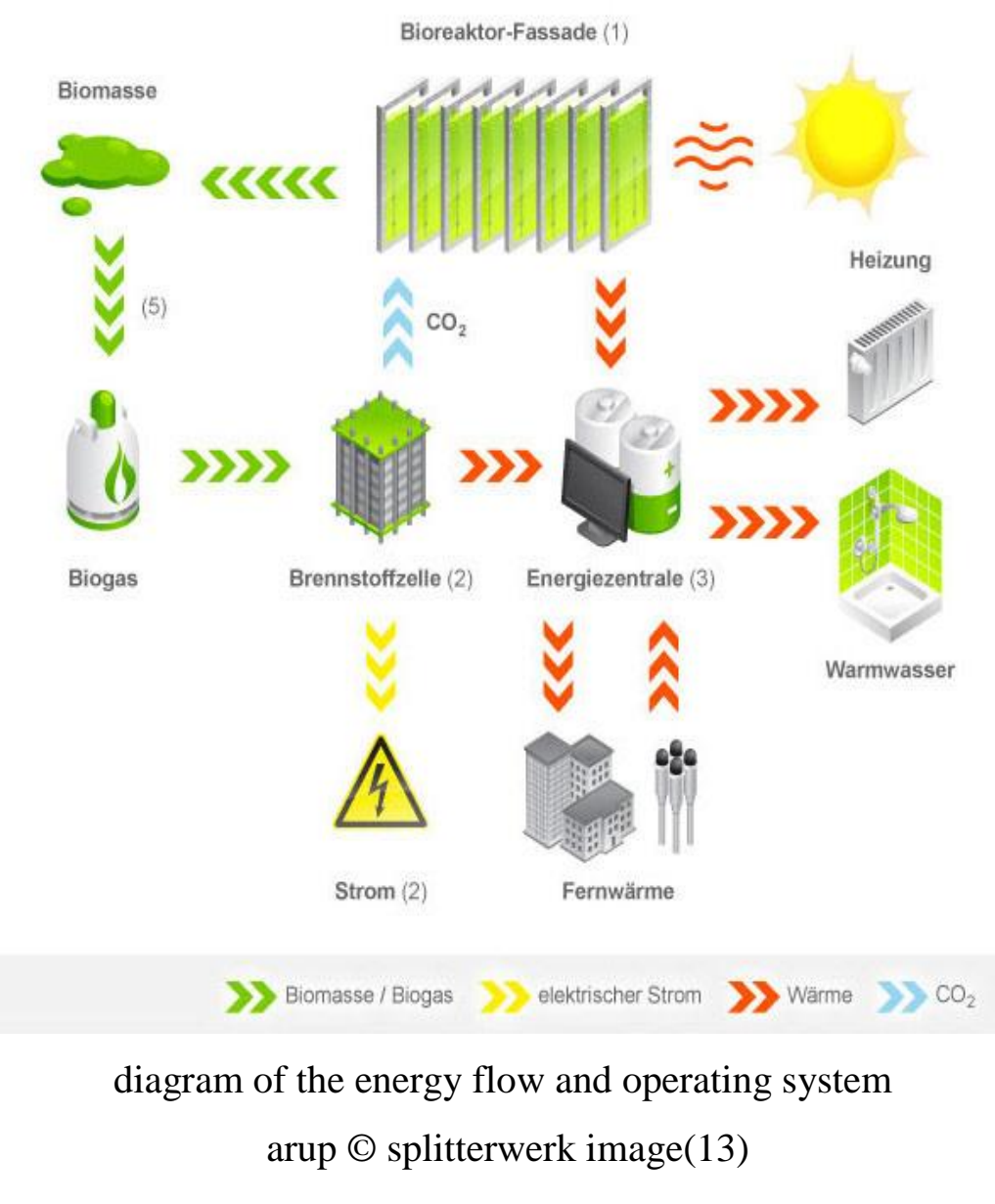

\section{Urban Algae Canopy Produces a Forest's Worth of Oxygen Daily}

Generating as much oxygen per day as 400,000 square feet of natural woodland, the Urban Algae Canopy combines architecture, biology and digital technology to create a structure that generates energy, responds to and enhances its environment Created by EcoLogics Studio and demonstrated in Milan, Italy, this "world's first biodigital canopy integrates micro-algal cultures and real time digital cultivation protocols on a unique architectural system,"with flows of water and energy regulated by weather patterns and visitor usage. Sun increases photosynthesis, for example, causing the structure to generate organic shade in realtime. In addition to $\mathrm{CO} 2$ reduction, the canopy as a whole can produce over 300 pounds of biomass daily, all through a relatively passive system that requires far less space and upkeep than conventional civic greenery. 


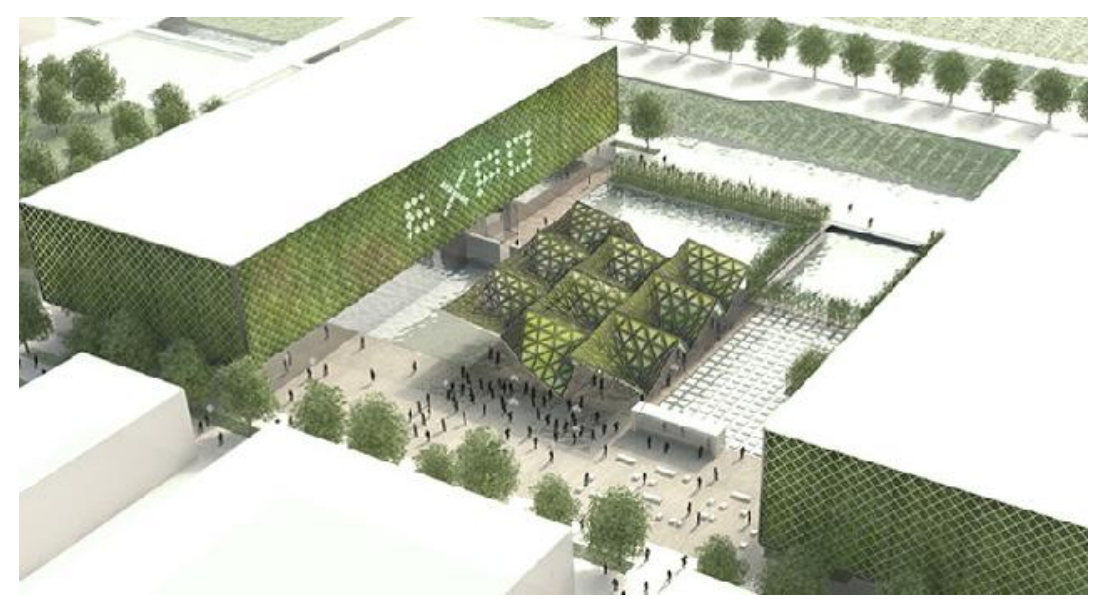

Design view of the canopy in the forest on a large scale (image14)

A hybrid of architectural and ecosystem design, the canopy is made to adapt its features based on manual as well as environmental inputs, letting users exert control (via a digital interface) within a larger dynamic system. "This process is driven by the biology of micoalgae is inherently responsive and adaptive; visitors will benefit from this natural shading property while being able to influence it in real-time." (image14)

For EcoLogics, this is only a step toward a larger vision - organic systems tied to high-tech ones in current and future buildings and infrastructure, as well as a breakdown of the differentiation between urban and rural, cities and nature.

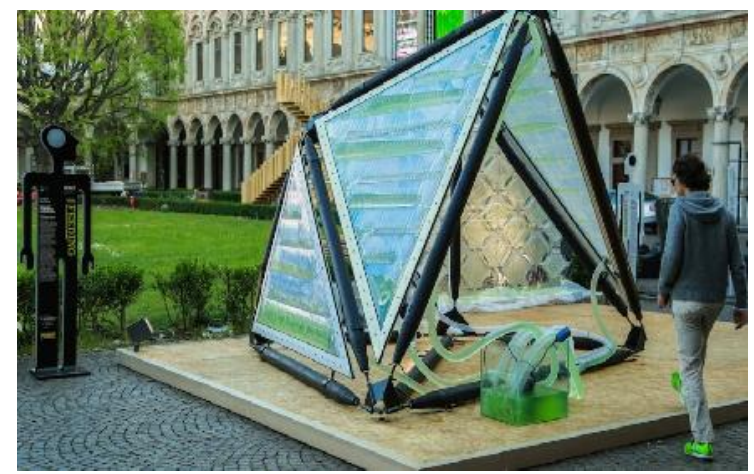

The algae of canopy at the day(image15)

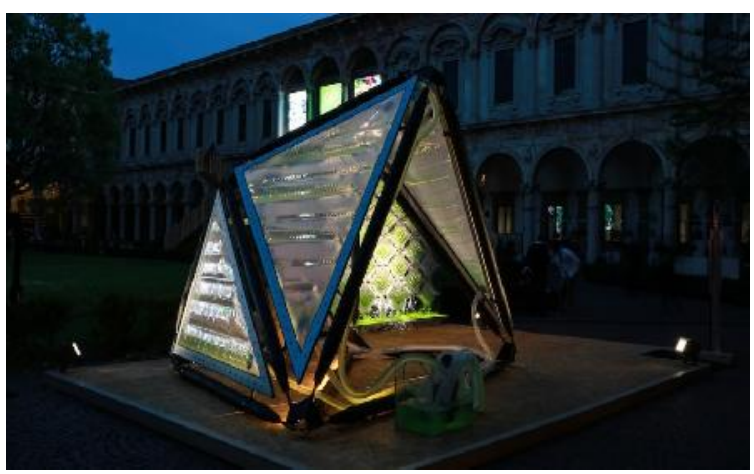

The algae of canopy at the night (image16) 
Indeed, the Canopy is just the latest of many projects the EcoLogics team has executed around similar themes, though it combines a series of construction techniques and materials they have been developing: "The exceptional properties of microalgae organisms are enhanced by their cultivation within a custom designed 3 layers ETFE cladding system. A special CNC welding technology is at the core of it and enables ecoLogicStudio to design and control the morphology of the cushions under stress as well as the fluid dynamic behaviour of the water medium as it travels through it."

Integrating organic and artificial systems opens up sustainable possibilities for everything from temperature control to power generation methods using advantages of both natural and digital parts. (image 15,16 ) It is also conceivable that the organic inputs and outputs of such systems could eventually be integrated into the production cycle of urban vertical farms. For now, the canopy will remain a working prototype and proof of concept as well as a chance to experiment with refining the constituent technologies. Meanwhile, others are taking different approaches to the use of algae in urban objects, including a design for smog-eating algae street lamps and this bio-voltaic table. (image17)

More from its creators: "In ecoLogicStudio we believe that it is now time to overcome the segregation between technology and nature typical of the mechanical age, to embrace a systemic understanding of architecture. In this prototype the boundaries between the material, spatial and technological dimensions have been carefully articulated to achieve efficiency, resilience and beauty." For an active application on a permanent building exterior with additional scientific details, be sure to take a look at the world's first bio-adaptive facade in Germany

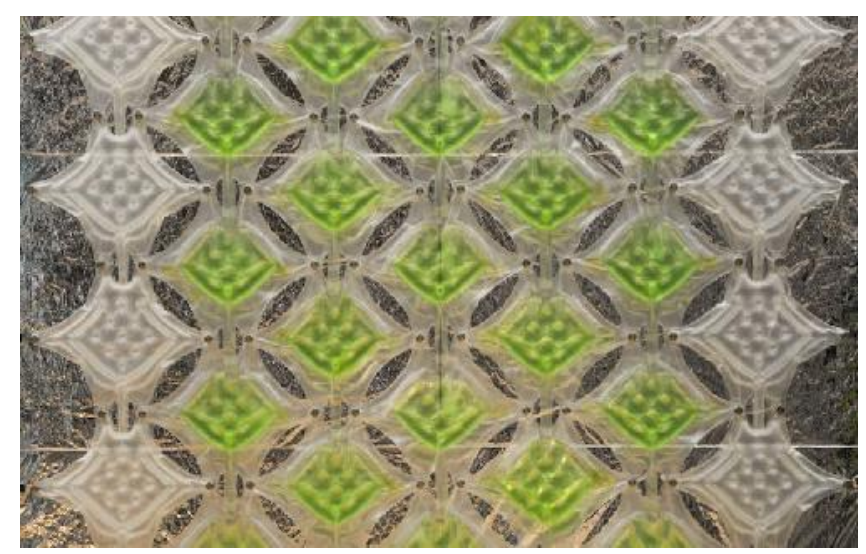

Algal biofuels in the near future (image17) 


\section{Algal biofuels in the near future}

In 2007 , the cost of a species of microalgae oil production capacity $30 \%$ fat by using closed photo-bioreactors with a capacity of 1,000 tons per year was estimated at approximately 5.10 dollars per gallon. Of course, this amount regardless of the costs associated with the conversion to bio-fuel oil, fuel and taxes were calculated shipping cost. However, at the same time fossil fuel cost per gallon was around $\$ 4$. Now the high cost of such fuels is the biggest challenge algal biofuel production. But in the next few years with the limited resources of fossil fuels and the increasing growth of prices on the one hand and by optimizing algal biofuel production processes on the other hand, the situation will change and soon the time will come when Biofuel algal will be affordable. (Amir Omidvar, 2014)

\section{CONCLUSION}

Architecture steps forward in recent years and continue using clean and renewable energy sources in urban areas, and residential buildings and other sciences through the use of more strides toward a better life pose. The View this type of architecture is not limited to residential buildings, and in the not too distant future in public buildings and high rises and even in open spaces and forests with new technologies proper design and effective performance will be provided. When several strains of studies from around the world in a matter very close to the same results is a sign of the word of knowledge throughout the world. Despite years of various specialties contribute to each other to achieve better results. Energy uses lies in algae and converts this energy into useful energy needed for the society of biologists and chemist's series of stories that attracted the first step.

What is certain is that researchers are still in the early stages of work and studies on energy issues algae, are the results of studies and experiences extraordinary steps must be taken to use public in general. One of the obstacles is the exorbitant cost of these projects. The thing that is important and it should be the basic principle for mankind named, maintaining a healthy environment for humans and the use of clean energy and renewable energy instead of fossil. Surely architects share in the operation and use of this energy is very important and necessary. 


\section{REFERENCES}

$1-\underline{\text { http://www.gizmag.com }}$

2 - http://www.designboom.com

3 - https://www.nextnature.net

4 - http://weburbanist.com

5 - http://inhabitat.com

6 - http://www:smart geometry.org

\section{7- http://www.fastcoexist.com}

8- http://www.sscs.co.th8- 8-

9-Essay "microalgae, future sources of bioenergy production" Amir Omidvar, the Journal of

Renewable and New Energy Forum, the first issue of Spring 2014

10- Essay "Nanotechnology and Sustainability" Mousavi, Noor Almahdy and Razavi, M.

Climate Conference, building and energy efficiency. 2011

\section{How to cite this article:}

Sepehri F. Lighting and energy supply for heating in building using algae power. J. Fundam. Appl. Sci., 2016, 8(3S), 1021-1036. 